\title{
Automatic Transition Prediction for Three-Dimensional Configurations with Focus on Industrial Application
}

\author{
Normann Krimmelbein* and Andreas Krumbein ${ }^{\dagger}$ \\ Deutsches Zentrum für Luft- und Raumfahrt - DLR (German Aerospace Center), Germany
}

\begin{abstract}
A computational method to predict transition lines for general three dimensional configurations is presented. The method consists of a coupled program system including a 3D Navier-Stokes solver, a transition prediction module, a boundary-layer code and a stability code. Focus is placed on the industrialization of the approach. For this, the transition prediction module has been adapted to be used for parallel computation to account for high computational demands for three dimensional configurations. Different calculation methods for the laminar boundary layer that are available in the transiton prediction module are presented. The method is validated against experimental data of the flow around an inclined prolate spheroid. Application examples are shown for different three-dimensional aircraft configurations and topics arising from these tests concerning the industrialization of the method are discussed.
\end{abstract}

\section{Introduction}

$\mathrm{T}$ accurately predict the aerodynamic performance of general aircraft configurations, the reliable prediction and modelling of the laminar-turbulent transition in Reynolds-averaged Navier-Stokes (RANS) solvers is often a necessary requirement. Hence, for the computation of flows around complex, threedimensional geometries in aerospace industries, a robust and automated transition prediction method is required. The transition from laminar to turbulent flow is a complex phenomenon and can occur through very different mechanisms depending on on-flow parameters such as Reynolds and Mach number or freestream turbulence, on surface properties or on the detailed development of the laminar boundary layer.

Existing transition prediction methods vary in their approaches from purely empirical transition criteria over physically based stability equations that take into account non-local and non-linear effects of disturbance growth, correlation based transition models ${ }^{1,2}$ to direct numerical simulations (DNS). The state-of-theart transition prediction method for thin aircraft boundary layers is the so called $e^{N}$-method, ${ }^{3,4}$ based on local, linear stability theory. $e^{N}$-methods were thoroughly calibrated and used in many applications of wing flows that are unstable due to Tollmien-Schlichting (TS) or cross flow (CF) instabilities ${ }^{5,6}$ and thus represent significant industrial value. Recent advances in predicting transition onset for complex flows address the prediction of unsteady transition on moving airfoils ${ }^{7}$ and the application to $2 \mathrm{D}$ laminar separation bubbles $^{8}$ including unsteadiness effects. ${ }^{9}$ Increasing focus is placed on the prediction of transition for threedimensional boundary layers on high aspect ratio wings and high lift configurations, ${ }^{10-13}$ flows around bodies of revolution ${ }^{14}$ and general three-dimensional aircraft configurations. ${ }^{15,16}$

The present work addresses the prediction of transition for flows around general three-dimensional, complex configurations. For automatic transition prediction in Navier-Stokes computations a transition prediction module is applied. The module uses different approaches to calculate the relevant data for transition prediction. To account for the computational effort for the three-dimensional flow calculation around general three-dimensional components and configurations, the transition module can be used in fully parallel

\footnotetext{
${ }^{*}$ Research Scientist, Institute of Aerodynamics and Flow Technology - $\mathrm{C}^{2} \mathrm{~A}^{2} \mathrm{~S}^{2} \mathrm{E}$ Center for Computer Applications in AeroSpace Science and Engineering, Lilienthalplatz 7, 38108 Braunschweig, Germany.

${ }^{\dagger}$ Research Scientist, R\&D Engineer, Institute of Aerodynamics and Flow Technology $-\mathrm{C}^{2} \mathrm{~A}^{2} \mathrm{~S}^{2} \mathrm{E}$ Center for Computer Applications in AeroSpace Science and Engineering, Bunsenstraße 10, 37073 Göttingen, Germany. AIAA senior member.
} 
Navier-Stokes computations. The objective of this paper is to give insight in the industrialization of the transition module, that is the developments improving the intensive use of the method in aerospace industry, for example for wing design.

\section{Description of methods}

For automatic transition prediction in Navier-Stokes computations a coupled program system has been developed (Fig. 1). This system consist of a transition prediction module ${ }^{12,16,27}$ implemented directly into the unstructured/hybrid RANS solver TAU of the German Aerospace Center (DLR). ${ }^{18,19}$ Specific elements of the module are the boundary-layer code $\mathrm{COCO}^{20}$ for swept, tapered wings and the linear stability equations solver LILO. ${ }^{21}$ The transition prediction module, which has been developed with special focus on predicting transition for flows around general complex, three-dimensional geometries supports parallel computing.

\section{A. Navier-Stokes solver}

The TAU code ${ }^{18,19}$ is a Navier-Stokes solver for the calculation of viscous and inviscid flows around general complex geometries. The solver is based on the finite volume method and uses a dual grid approach where the flow variables are associated with the vertices of the original grid.

The TAU code is an unstructured solver that can handle different cell types and hence can be used on structured and unstructured (hybrid) grids. Generally, a semi-structured grid layer above surfaces is used to resolve boundary layers, whereas the rest of the computational domain is filled with an unstructured grid.

For parallel computations, a domain decomposition approach is used and the massage passing concept using $\mathrm{MPI}^{22}$ is applied. In a preprocessing step, the grid is divided into a certain number of subdomains using a bisection algorithm. ${ }^{19}$ After partitioning the grid, the solver computes the flow data on a single domain per process. The data is regularly updated on points lying in the overlap region between a certain domain and its neighbours.

The solver computes the fluxes with a second-order central scheme or one of various upwind schemes with linear reconstruction for second-order accuracy. Time integration is per-

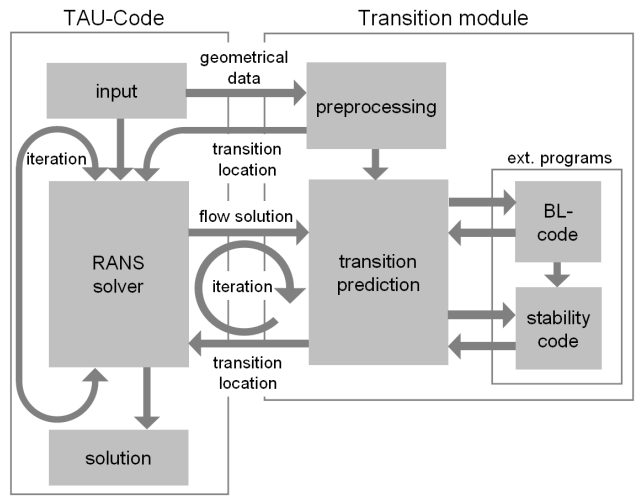

Figure 1. Coupled program system. NavierStokes solver TAU with transition module. formed by either applying an explicit, multistage Runge-Kutta scheme or an implicit, lower-upper symmetric Gauss-Seidel (LU-SGS) scheme. Turbulent flows are modelled using different Spalart-Allmaras, k- $\omega$ models or Reynolds stress turbulence models. For transitional flows, laminar regions can be designated by the definition of polygon lines on the surface of the geometry and prescribing the maximum height of the laminar region over the surface. For these computations, the turbulent production terms are suppressed in the laminar flow area.

For convergence acceleration residual smoothing, local time stepping and a multigrid approach can be applied. To extend the solver capability to incompressible flows, a low-Mach-number preconditioning approach is implemented.

\section{B. Transition prediction method}

The classical linear stability theory evaluates the stability of a laminar boundary layer by examining the development of small disturbances. This is used for transition prediction in form of the $e^{N}$-method. The principal approach of the theory is to superimpose instationary disturbances onto the stationary boundarylayer flow. The assumption of a harmonic wave as disturbance is superposed to the mean flow quantities:

$$
q^{\prime}(x, y, z, t)=q(z) e^{(\alpha x+\beta y-\omega t)}
$$

This approach leads to a system of second-order differential equations, which form a linear eigenvalue problem for the complex disturbance frequency $\omega$. That is, the temporal stability problem is considered 
and the complex wave numbers $\alpha$ and $\beta$ have to be prescribed. The parameters of the eigenvalue problem depend on the basic flow properties $(M a, R e)$, on the local velocity and temperature profiles of the laminar boundary layer and on their first and second derivatives. Generally, the boundary-layer flow is assumed to be a parallel flow. ${ }^{23}$

The solution of the eigenvalue problem results in local amplification rates, which are integrated along an appropriate integration path. ${ }^{23}$ For this, the Gaster transformation ${ }^{24}$ is used, to transform the temporal growth into spatial growth. The velocity and direction of the energy transport of a wave is represented by the group velocity and thus, the group velocity can be taken as the amplification direction. ${ }^{23}$ The integration path for the local amplification rates is then given by the trajectory of the group velocity.

Different integration strategies for the computation of the $N$-factor from the amplification rates are available. ${ }^{23}$ In the coupling with the transition module described in this paper, the prescribed-frequency/prescribed-propagation-direction integration strategy ${ }^{23}$ is used for Tollmien-Schlichting instabilities, and the prescribed-frequency/prescribed-wavelength integration strategy ${ }^{23}$ is used for cross flow instabilities.

\section{Application of the transition prediction method in $3 \mathrm{D}$}

\section{Determination of boundary-layer data}

It can be shown, that for general transition prediction, and especially for the prediction of cross flow type transition, a high normal-to-wall mesh density is required to resolve the boundary layer adequately. ${ }^{16}$ To overcome this constraint, a boundary-layer method is in many cases an efficient alternative to a high resolution Navier-Stokes computation. However, boundary-layer methods, and in particular quasi-2D boundary-layer methods, have certain limitations for complex flows, for example for flows around complex geometries and low aspect-ratio wings or in the presence of laminar separation bubbles. To deal with both, the use of coarse grids across boundary layers for rapid engineering applications and highly resolved boundary layers for detailed flow analysis, a hybrid approach is used here, that is boundary-layer data can either be extracted directly from the Navier-Stokes solution or a first-order boundary-layer method for swept, tapered wings ${ }^{20}$ using the conical flow assumption ${ }^{25}$ is applied.

In the presented method, boundary-layer data are calculated along two different types of lines (hereafter generally referred to as 'transition prediction lines'), depending on the method to calculate the velocity profiles (figure 2): streamlines along the boundary-layer edge for the extraction of boundarylayer data from the Navier-Stokes solution and line-in-flight cuts of the geometry for the application of the boundary-layer method. (It has to be noted that the boundary-layer edge is not a streamline. Referring to edge streamline in this context means the "local projections of the loci, where the streamlines cross the boundary-layer edge". ${ }^{26}$ )

The calculation of the edge streamlines is based on a multistage Runge-Kutta scheme and uses the boundary-layer edge velocities, projected onto the geometry's surface, for integration. After the calculation of the 3D edge streamlines, the velocity profiles along the streamlines are extracted directly from the Navier-Stokes solution and are used for the transition prediction.

The boundary-layer code is applied to line-in-flight cuts distributed along the wing span. These cutting lines are the intersections of the surface of the geometry with planes parallel to the on-flow direction. During the runtime, the pressure distribution along the line-in-flight cuts is interpolated from the Navier-Stokes solution and is used as input for the boundarylayer method. The boundary-layer code returns highly accurate laminar boundary-layer profiles for transition prediction.

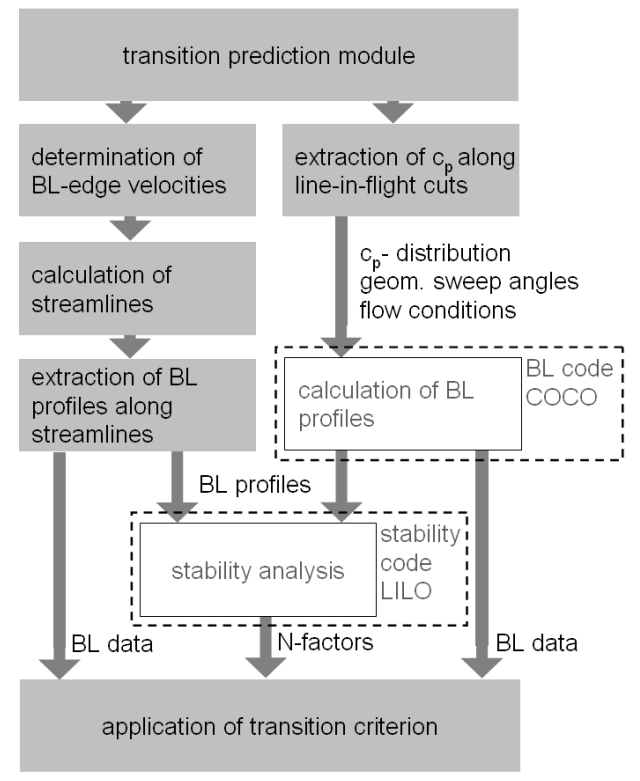

Figure 2. Different approaches to calculate laminar boundary-layer data. 


\section{Application of the $e^{N}$-method}

If the $e^{N}$-method is used for transition prediction, a suitable integration path to calculate the $N$-factors from the amplification rates is the group velocity trajectory (section B).

The two external programs, the boundary-layer method COCO and the stability solver LILO, form a coupled program system to evaluate the $N$-factor distribution along line-in-flight cuts for swept, tapered wings. Consequently, they are based on the same leading edge oriented coordinate system, which is eventually employed when the $N$-factors are calculated by the stability solver. Moreover, the underlying conical flow assumption allows for the simple calculation of the group velocity trajectory for the integration of the amplification rates. Hence, when using the line-in-flight cuts together with the boundary-layer method, the calculated $N$-factors are taken directly from the stability solver and are used for application of the $e^{N}$-method. For a general three dimensional approach (when using the edge streamlines), the calculation of the group velocity trajectory is not as straigth forward. Instead, the following simplification is made: The group velocity trajectory is approximated by an edge streamline ${ }^{23}$ and this streamline is used as the integration path. For this, only the local amplification rates are taken from the stability code and are seperately integrated along the previously calculated edge streamlines.

Whatever method is used, the integrated amplifications yield a single $N$-factor curve for a single frequency/wavelength. To cover the whole spectrum of amplified waves, integrations for different frequencies/wavelengths are performed. The envelope of the $N$-factor curves then represents the maximum amplification and the envelope is analysed in the transition prediction module with appropriate $N$-factor criteria to give new transition locations for every streamline/line-in-flight $\mathrm{cu}$. The limiting $N$-factors are applied using the $2 N$-factor strategy, treating the $N$-factors for Tollmien-Schlichting and cross flow instabilities independently. The interaction of Tollmien-Schlichting and cross flow waves can not be evaluated by linear local stability theory and is instead modelled by applying a stability curve, where the critical $N$-factor of one instability form depends on the local $N$-factor of the other instability form. ${ }^{14}$

\section{Industrial application}

In aerospace industry, there exists the general demand for a transition prediction method, for example for the design process of wings. An important requierement of such a method is an automatic and autonomous application to general 3D flows around complex configurations. Also, the regular use of an automatic transition prediction functionality in an industrial environment reveals the need for robustness and user friendliness. Now, that the verification and validation of the $e^{N}$-method to general three dimensional flows has remarkably advanced over the past years and that the $e^{N}$-method has gained general acceptance in aerospace industry, growing focus has to be placed on the industrialization of an automatic application of the methodology. ${ }^{27,28}$

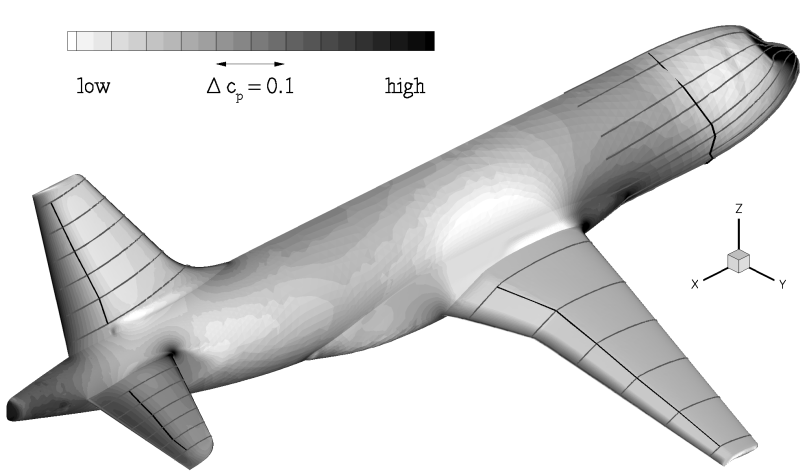

Figure 3. Edge streamlines and transition locations. Generic transport aircraft, $\alpha=-4.0^{\circ}, M a=0.2, R e=$ $2.3 \times 10^{6}, i_{H}=4.0^{\circ}$.

tail planes and fuselage, but also include for example nacelles.

The computational capacities in research and industry are generally growing and hence allow for higher resolutions of the computational grid around three dimensional configurations at reasonable computing times. However, the resolution of the computational grids commonly used is still far away to capture all flow features necessary for accurate transition prediciton, for example the cross flow components of the velocity profiles inside the boundary layer. This generally means that the state of the art for transition prediction with the $e^{N}$-method is the use of a boundary-layer method to generate highly accurate boundary-layer data.

The main target of the industrialization of the transition prediction module is to robustly, accurately and efficiently predict transition on all relevant aerodynamic surfaces of a general transport aircraft. Relevant aerodynamic surfaces are wing,

The prediction of transition lines for wing, vertical and horizontal tailplane and fuselage of a transport 
aircraft is generally feasible with the presented method if the boundary-layer data for all non-wing-like surfaces is extracted from the Navier-Stokes solution. ${ }^{16,29}$ Figure 3 shows a generic transport aircraft at $\alpha=-4.0^{\circ}, i_{H}=4.0^{\circ}, R e=2.3 \times 10^{6}, M a=0.2$ for which the presented method has been applied. The computational grid has a moderate resolution of the boundary layer, with 32 grid points normal to the wall, except for the horizontal tail plane with 48 points normal to the wall, giving a total number of grid points of 12 million. The chosen grid density in the boundary layer results in a fairly accurate prediction of boundarylayer profiles while keeping the overall computational demand low. However, not for all surfaces the linear stabililty theory was used. For the fuselage a simple transition criterion was applied, setting the transition a short distance downstream of the pressure minimum. The main wing is equipped with a deflected flap, leading to large separated areas at the suction side well before transition would have been predicted by linear stability theory. Here, the laminar separation was used as transition point instead, in order to avoid unsteadiness effects and convergence problems during the Navier-Stokes iterations.

For all other surfaces (wing pressure side, horizontal and vertical tailplane) linear stability theory in form of the $e^{N_{-}}$ method with a critical $N$-factor of 7.5 was applied. With regard to the moderate resolution of the boundary layers, only Tollmien-Schlichting instabilities were considered. Previous investigations indicated that a much higher grid resolution is needed for accurate prediction of cross flow instabilities. ${ }^{16,29}$ The case was run completely in parallel mode, using a partitioning of 8 domains for the grid. For faster convergence, low-Mach-number preconditioning was applied, together with the implicit LU-SGS time integration scheme. Turbulent flow was modelled using the standard Spalart-Allmaras turbulence model. Figure 3 shows the calculated edge streamlines together with the converged transition lines. For all wing like surfaces 6 streamlines have been considered to resolve the problem, the fuselage has been covered with 11 streamlines. The predicted transition lines are located in the adverse pressure gradient region, as expected for the investigated transition scenario.

This test shows the ability of the transition prediction module to predict transition on all relevant transitional surfaces of a

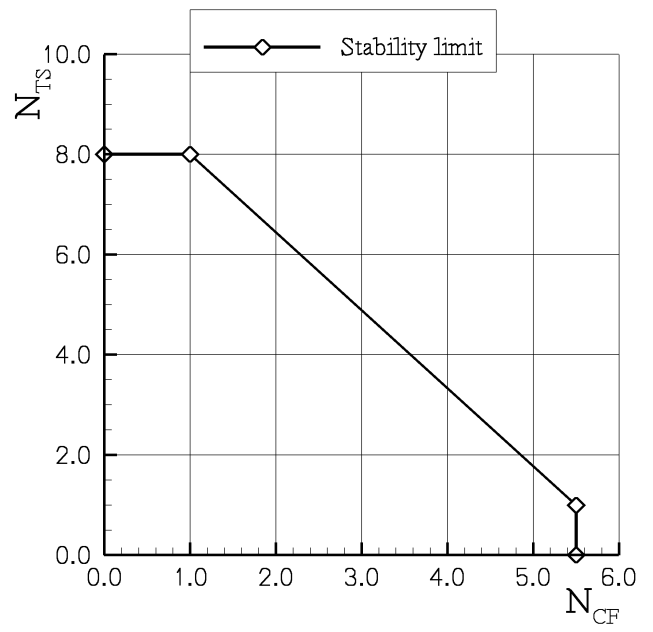

Figure 4. Stability diagram. $N_{T S}$ vs. $N_{C F}$. generic transport aircraft configuration. It is especially demonstrated that the approach using edge streamlines from the Navier-Stokes solution is suitable for transition prediction for geometrically very different components (fuselage, wing).

\section{A. Validation of the method}

The validation of the transition prediction method is a necessary and important part of the utilization of this method in industrial applications. Much progress has been achieved in the application and validation of the $e^{N}$-method to high aspect ratio wings. ${ }^{27,28,30}$ Especially the combination of linear stability theory together with an application of a boundary-layer method along line-in-flight cuts is nowadays widely used. However, the database of validation cases for general, three-dimensional transitional flows is yet limited. One such test case is the flow around an inclined 6:1 prolate spheroid, for which comprehensive measurements of the flow were accomplished at the DFVLR (now DLR) $3 \mathrm{~m}$ x $3 \mathrm{~m}$ low speed wind tunnel Göttingen. ${ }^{31,32}$ Surface hot film probes measuring the local wall shear stress were applied for the investigation of the threedimensional boundary layer. 12 measuring stations in streamwies direction and 30-80 measuring stations in circumferencal direction were used. The evaluation of the local wall shear stress provides detailed information of the laminar-turbulent transition of the boundary layer. The measurements include various Reynolds numbers and angles of attack. At certain on-flow conditions, transition is characterized for this case to change from pure Tollmien-Schlichting transition to pure cross flow transition with regions where both types of waves may interact and lead to transition.

It is well known that local, linear stability theory can not analyse the interaction of Tollmien-Schlichting and cross flow waves from first principles. However, an empirical approach to overcome this deficiency is to reduce the critical $N$-values in the $N_{T S}-N_{C F}$ space for simultaneously excited Tollmien-Schlichting and cross flow waves by assuming that the critical $N_{T S}$-factor decays linearly with increasing $N_{C F}$. Numerical 


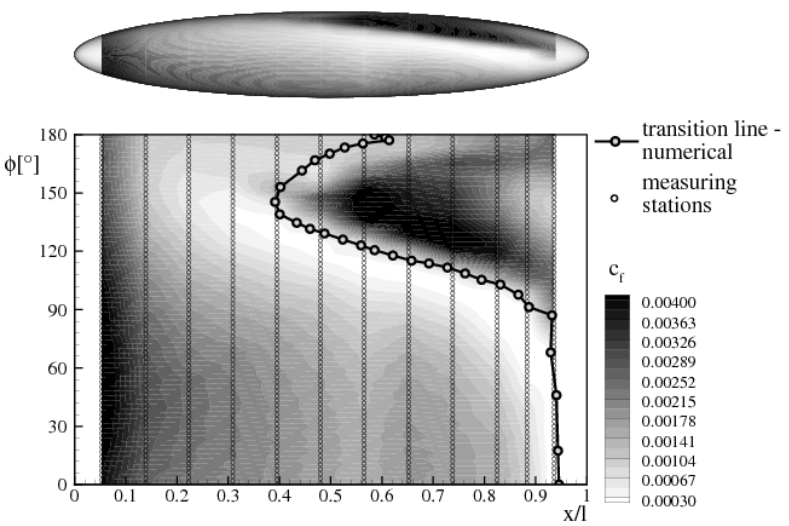

(a) $\alpha=10.0^{\circ}, R e=1.5 \times 10^{6}, M a=0.03$, experimental
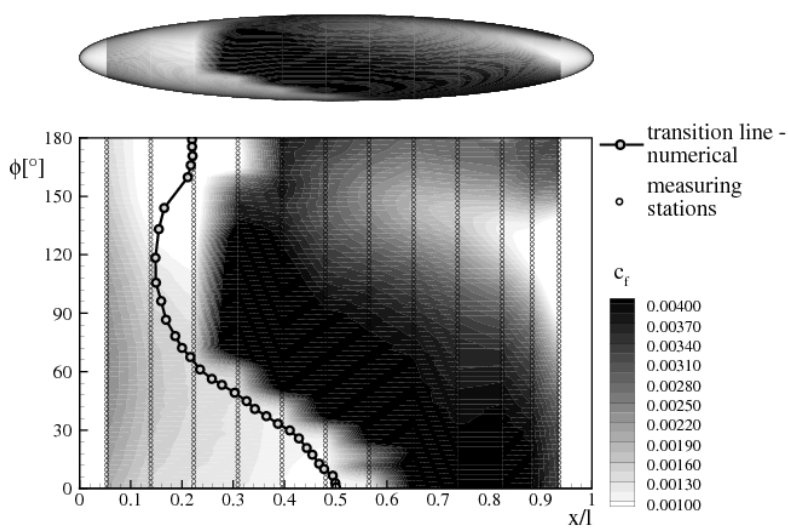

(c) $\alpha=10.0^{\circ}, R e=6.5 \times 10^{6}, M a=0.13$, experimental

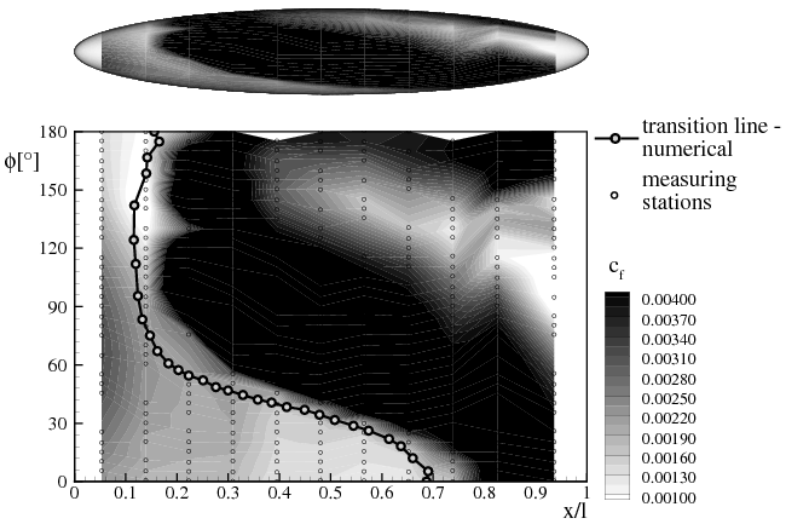

(e) $\alpha=15.0^{\circ}, R e=6.5 \times 10^{6}, M a=0.13$, experimental

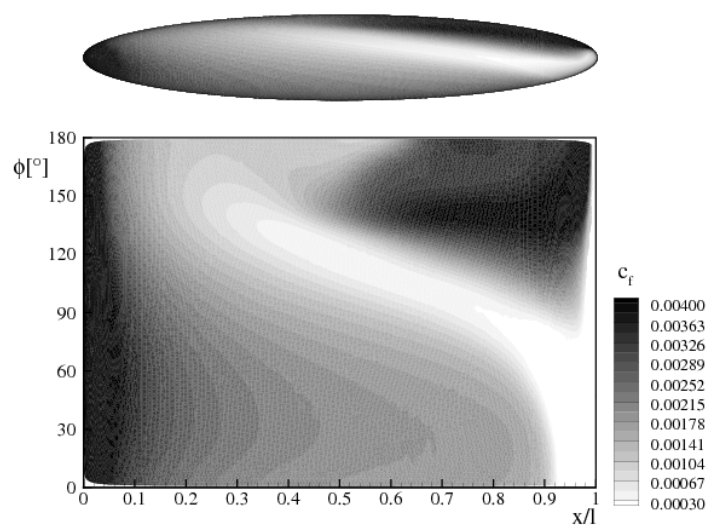

(b) $\alpha=10.0^{\circ}, R e=1.5 \times 10^{6}, M a=0.03$, numerical

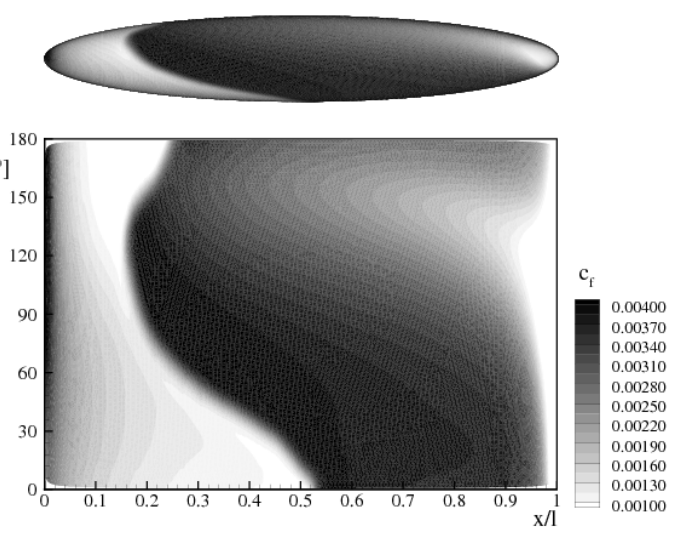

(d) $\alpha=10.0^{\circ}, R e=6.5 \times 10^{6}, M a=0.13$, numerical

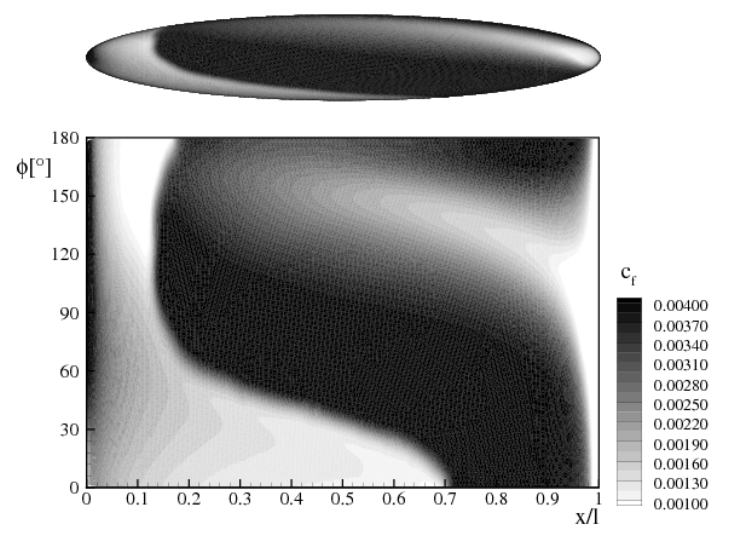

(f) $\alpha=15.0^{\circ}, R e=6.5 \times 10^{6}, M a=0.13$, numerical

Figure 5. Comparison of measured and computed skin friction distribution and transition locations. 6:1 prolate spheroid 
investigations of the transition for the flow around the inclined prolate spheroid in ${ }^{14}$ yield the diagram of figure 4, which was applied for the present validation calculations to account for the interaction of the two wave types.

The steady calculations were carried out using low-Mach-number preconditioning and the implicit LUSGS time integration scheme. Turbulent flow was modelled using the standard Spalart-Allmaras turbulence model. For the boundary layer a normal-to-wall resolution of 128 points was used, that is $60-100$ points resolve the laminar boundary layer of the prolate spheroid. The resolution in streamwise direction is approx. 300 points, the overall number of grid points is 2.8 million. On-flow conditions varied from $M a=0.03$ to $M a=0.13, \alpha=10.0^{\circ}$ to $\alpha=15.0^{\circ}$ and $R e=1.5 \times 10^{6}$ to $R e=6.5 \times 10^{6}$. For the transition prediction 31 streamlines were equally distributed over the prolate spheroid's surface.

The comparison between experimental and numerical results has been made by plotting the numerical transition line onto the contour plot of the experimental wall shear stress. From the local increase in wall shear stress, transition on the prolate spheroid can be estimated. For an improved visualization of the overall information of the transition, the surface of the prolate spheroid has been mapped onto a $2 \mathrm{D}$ plot. The measuring stations from the experiment are given in the contour plots as small circles. As reference, the wall shear stress distribution of the Navier-Stokes computation is plotted too (figure 5).

For the low Reynolds number case $\left(R e=1.5 \times 10^{6}, M a=\right.$ $\left.0.03, \alpha=10.0^{\circ}\right)$, transition is triggered purely by TollmienSchlichting instabilities and is very well predicted by the transition module (figure 5(a)). Although for this case cross flow amplifications are present during the iterative transition prediction, the final, converged transition is purely caused by Tollmien-Schlichting instabilities.

Generally for all cases, transition is caused by TollmienSchlichting waves near the windward and leeward symmetry lines of the prolate spheroid, where the flow is more twodimensional. However, regarding the high Reynolds number

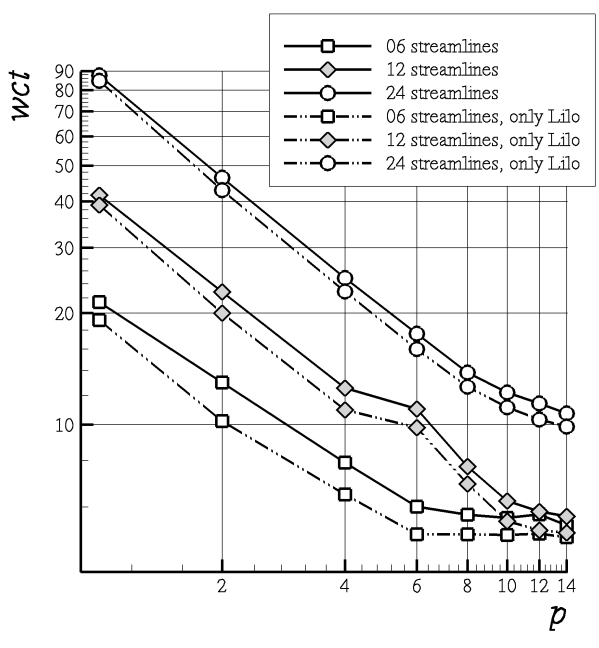

Figure 6. Computational time wct vs. number of processes $p$. Transition prediction step and execution of stability code. 6, 12 and 24 streamlines. cases $\left(R e=6.5 \times 10^{6}, M a=0.13, \alpha=10.0^{\circ}\right.$ and $\left.15.0^{\circ}\right)$, cross flow instabilities play a growing role in the transition process. For $\alpha=10.0^{\circ}$ (figure $5(\mathrm{c})$ ), a significant portion of the transition line is represented by simultaneously excited Tollmien-Schlichting and cross flow waves and regions with pure cross flow transition. Increasing the angle of attack to $15.0^{\circ}$ (figure 5(e)) leads to the development of a large region with pure cross flow transition. For both cases, transition is predicted slightly too far upstream but is still in good accordance with the experiment.

\section{B. Parallelization and computational performance}

Parallelization by means of the transition prediction module is needed for the determination of wall-normal lines, the assembly of velocity and temperature profiles along these normals, the calculation of edge streamlines, the assembly of line-in-flight cuts and the execution of the sequential, external programs.

The TAU code uses an domain decomposition approach for parallel computation. For a given number $p$ of processors the computational grid is divided into $p$ subgrids (subdomains). Each of the processors computes on one of the subgrids. A continuous communication between the processes is then performed during the solution process by exchanging regularly local informations (flow quantities) at the domain boundaries. In contrast to this, for the transition prediction non-local data have to be communicated.

Calculation of data in form of lines within the transition module (that is wall-normal lines, edge streamlines, line-in-flight cuts) is effectively an ordered assembly of a list of grid points. These points are gathered, beginning at starting points (that is surface points, user-defined starting points) and ending at user-defined or geometrically provided endpoints. For parallel computation, another limit is a domain boundary. In this case, the endpoints of the lines will be communicated to the neighbour domain where they serve as new start points for another loop of the assembly of the lines. Finally, data from each line have to be assembled to 
obtain complete boundary-layer profiles and complete streamline/line-in-flight cuts for further treatment by the transition prediction module. ${ }^{29}$

Another parallelization strategy is applied for the external programs used by the transition module. These sequential programs are accessible from the transition module via file I/O and system calls and are not explicitly written for the utilization by a parallel and automated transition prediction process within a Navier-Stokes solver. Generally, the external programs are designed to process data of a single transition detection line (edge streamline or line-in-flight cut) at one sequential run. During a parallel computation, for each process of the Navier-Stokes calculation an external program is executed sequentially: if the NavierStokes calculation is run on $p$ processors, the external programs are started from each of the $p$ processes independently. Taking the stability analysis as example, this means, that $p$ stability analyses with the external stabiliy code can run parallel on the $p$ processes, so that $p$ transition detection lines can be processed parallel by the external program and a parallel performance of the actually sequential program is achieved. The advantage of this approach is that only few modifications of the source codes of the external programs had to be made. A disadvantage is that no full parallelization is obtained, if the number of streamlines is not an integer multiple of the number of processes.

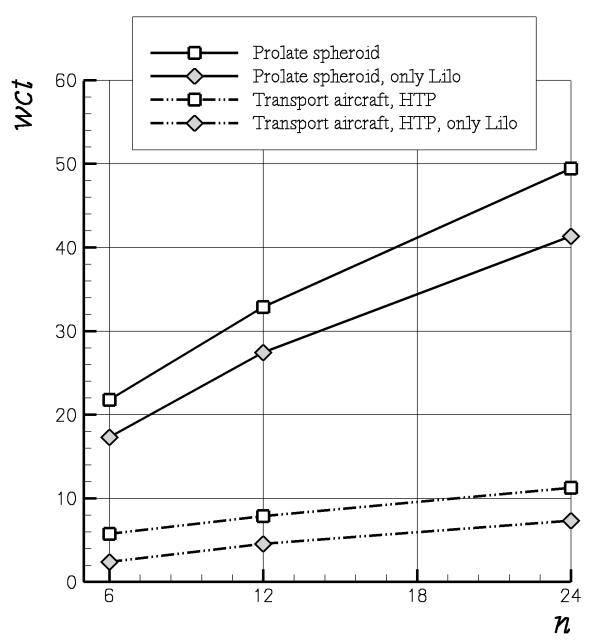

Figure 7. Computational time wct vs. number of streamlines $n$. 6:1 prolate spheroid, generic transport aircraft, 8 domains.

The main reason for the relatively high co the ratio of the average number of points on the streamlines to the overall number of grid points is rather high for this case. This means high computational effort for the stability analysis compared to the effort of the Navier-Stokes iteration. When comparing the computational time for the transition prediciton on the horizontal tailplane of a generic transport aircraft (figure 3) to the computational time for the prolate spheroid it can be seen, the the relative computational demand is lowered (figure 7). For both test cases only TollmienSchlichting instabilities are considered. The computational time is normalized with the corresponding time used for one Navier-Stokes iteration. For cases with a more favourable ratio of streamline size to grid size a significant reduction in relative computational time is obviously achieved. It has to be noted that the computational effort of the stability solver does not depend on the resolution of the boundary-layer profiles in the Navier-Stokes solver or the boundary-layer code. The input boundary-layer profiles are interpolated inside the stability code using cubic splines with certain a number of points that is independent of the input profiles.

Parallelization regarding the transition prediction module is here mainly considered as the ability to process partitioned Navier-Stokes solutions, a full parallel execution is in general not possible. For example, for the calculation of wall-normal lines, edge streamlines or line-in-flight cuts only domains containing sections of these lines are involved in the computation. Domains not containing sections of these lines have to run idle during this calculation process. This makes clear, that a complete parallel execution is not possible for all cases. 
The high computational effort of one transition prediction step compared to one multigrid cycle is relativized when regarding at the overall computational effort of a complete, fully converged calculation with transition prediction. The main part of the computational time is due to the iterative procedure of the transition prediction (figure 1). The high overall computational cost comes from rather large numbers of multigrid cycles needed to get converged intermediate solutions after updating the transition location. For the flow around the prolate spheroid (subsection A), an average overall number of time-steps of 20.000 35.000 was needed, with 4-6 transition prediction steps to reach a well converged solution. The fraction of the overall consumed time used by the transition prediction process was then approximately $1 \%$.

\section{Application to transport aircrafts}

\section{1. $3 D$ high lift configuration}

The transition prediction module was applied to a generic three-dimensional aircraft configuration. ${ }^{33}$ The geometry represents a modern transport aircraft with fuselage and a high lift wing system with a slat and a flap which is split into an inner and an outer part (figure 8). The flow conditions for this case are $\alpha=14.0^{\circ}$ at an free stream velocity of $u_{\infty}=60 \frac{\mathrm{m}}{\mathrm{s}}$ giving a Reeynolds number $R e=1.435 \times 10^{6}$ based on a reference length of $l_{\mu}=0.41 \mathrm{~m}$.

Transition has been predicted on all wing surfaces using the pressure distribution and local sweep angles and applying a boundary-layer code along line-in-flight cuts to generate the velocity profiles. The stability analysis was applied at 138 line-inflight cuts for the slat, 148 line-in-flight cuts for the wing, 29 line-in-flight cuts for the inboard flap and 73 line-in-flight cuts for outboard flap. These, in total 388, line-in-flight cuts are shown in figure 9 (a). At regions of geometrical discontinuities the calculation of line-in-flight cuts has been omitted. Depending on the degree of the discontinuity, these surface imperfections can lead to uncertainties in the determination of the sweep angles or on the smoothness of the pressure distribution which is used as input for the boundary-layer method. For the lower surface of the slat and the flap fully laminar flow was

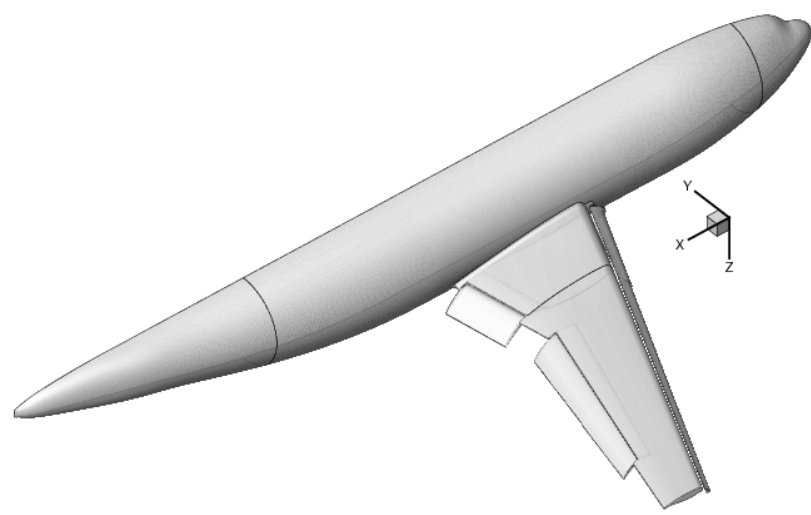

Figure 8. Geometry of 3D high lift configuration. assumed.

For the parallel computation on 96 processes, the overall number of 536 transition prediction lines (138 for the flap, 148 for the wing upper surface, 148 for the wing lower surface, 29 and 73 for the flap) is split to 5-6 transition prediction lines per process, according to the parallelization approach discussed in scetion B. This means, for each process 5-6 boundary-layer computations and 5-6 stability analyses with the external programs are performed for each transition prediction step.

For the upper surfaces of slat, wing and flap the predicted transition lines are shown in figure 9(a). They generally lie in regions of strong adverse pressure gradients (figure 9(b)), indicating transition due to Tollmien-Schlichting instabilities. A closer analysis of the transition lines reveals a rather smooth distribution in spanwise direction for the slat and flap. Minor jumps in the transition positions to locations close to the leading edges for slat and flap are caused by unevennesses in the pressure distribution which had an influence of the separation behaviour of the boundary-layer code.

A similar behaviour can be observed for the transition line of the wing suction side as well, however to a much larger extent. From one line-in-flight cut to the next, the transition point obviously jumps from a position well downstream of the pressure minimum to a position close to the leading edge. This results in a zig-zag-pattern in the transition line at the outboard part of the wing $(\eta=0.8-1.0$, figure 9 (a)). A closer look to the converged flow solution reveals a well resolved suction peak near the leading edge of the wing. If looking at a slice of the flow field at a spanwise position of $\eta=0.9$, this phenomenon can be clearly visualized by regarding the contour plot of the pressure distribution (figure 10).

The flow exhibits a favorable pressure gradient from the pressure maximum at the attachment line to the leading edge of the wing, ending in a relatively small suction peak. After that, the flow is decelarated 


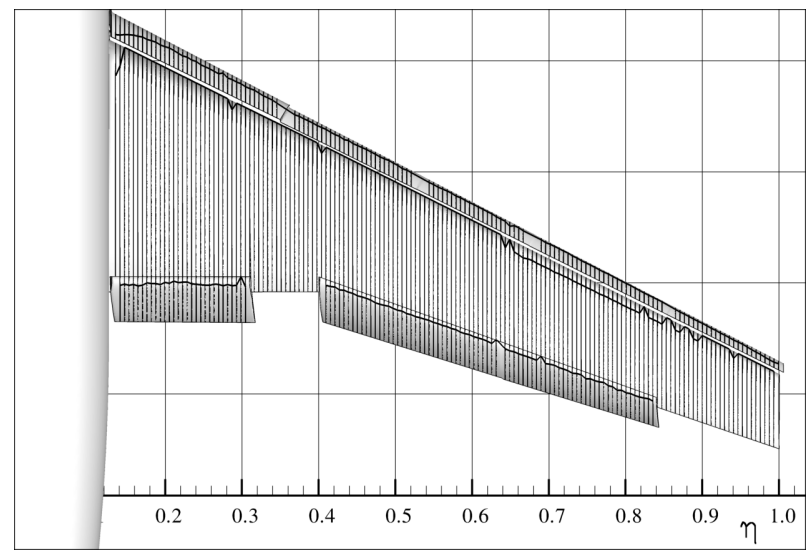

(a) Predicted transition lines and line-in-flight cuts used for transition prediction.

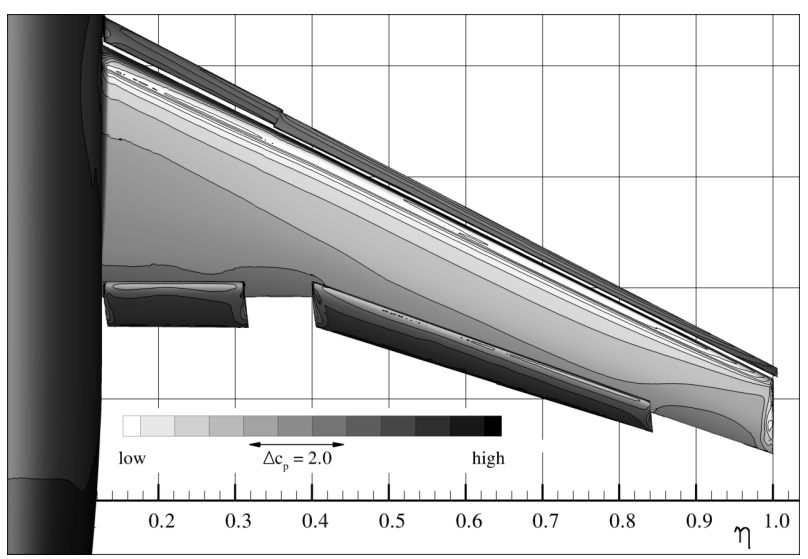

(b) Pressure distribution.

Figure 9. Upper surfaces of 3D high-lift configuration.

to a local pressure maximum until another strong accelaration of the flow leads to the formation of a strong suction peak on the wing's upper surface. This flow behaviour can be observed for the whole spanwise extend of the wing. Transition for this case is predicted either a very short distance downstream of the first small suction peak or downstream of the main suction peak.

The reason for this behaviour of the transition prediction method is a combination of the flow physics and the chosen approach for transition prediction, that is to use a boundary-layer method to calculate the input velocity profiles for the stability analysis. In this approach, transition can only be predicted upstream of the last point of the boundary-layer calculation of the boundary-layer code. It is obvious, that boundary-layer data are only available upstream of this point. Since the underlying boundary-layer equations become singular at a separation point, the laminar boundary-layer calculation within the boundary-layer code terminates when this point is reached. The detected laminar separation inside the laminar boundarylayer code is mainly driven by the input pressure distribution. In regions of adverse pressure gradient the flow decelerates and eventually separates, if the deceleration is strong enough. If no

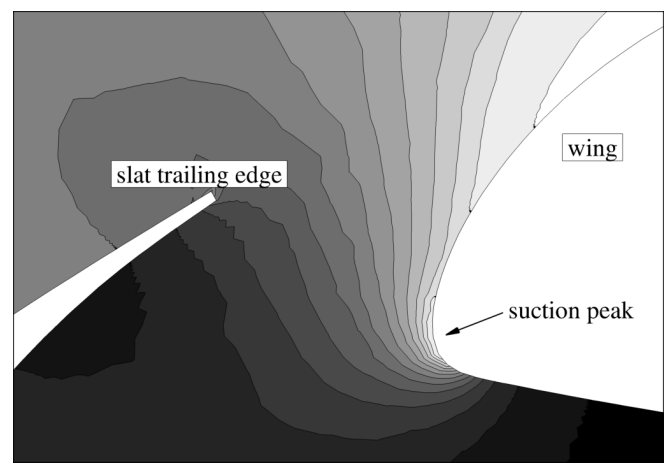

Figure 10. Pressure distribution near the wing leading edge for a slice at $\eta=0.9$. transition is predicted by the stability theory upstream of the separation point, the separation point itself is then used as transition position in the transition prediction process..

From this explanation the behaviour of the transition prediction becomes clearer: depending on the local resolution of the first small suction peak and the strength of the pressure gradient the boundary-layer code predicts a laminar separation close to the leading edge of the wing and transition is set far upstream. For some portions of the wing, the deceleration of the flow is not strong enough to cause early separation and transition is then predicted well downstream of the leading edge on the upper surface of the wing. It has to be noted: the small suction peak near the leading edge appears for the whole spanwise extend of the wing. However, no separation can be seen in the Navier-Stokes solution.

This phenomenon has been observed for different high-lift configurations that have been investigated so far by the authors. In particular, this phenomenon occured for high-lift configurations not operating at or near their design points (at maximum lift).

To further verify this observation, a small test series was carried out, using a $2 \mathrm{D}$ high-lift configuration. This 2D configuration consists of the A310 take-off configuration ${ }^{34}$ with slat, main airfoil and flap at $R e=$ $6.11 \times 10^{6}$ and $M a=0.221$. Investigations for this configuration are typically conducted at an angle of attack of $\alpha=21.4^{\circ} .{ }^{12}$ However, for this test series angles of attack of $\alpha=21.4^{\circ}, 15.0^{\circ}, 10.0^{\circ}, 5.0^{\circ}$ and 
$0.0^{\circ}$ have been investigated with particular focus on the flow behaviour at the leading edge of the main airfoil. A rather fine computational grid was used to avoid an influence of a badly resolved surface on the development of the pressure distribution. The grid has about 122,000 points and was designed to resolve laminar separation bubbles developing on the upper surfaces. ${ }^{12}$

It can be seen from the pressure distribution of the main airfoil in figure 11(a) that with decreasing angle of attack a suction peak near the leading edge starts to develop. This suction peak is further enlarged for lower angles of attack. For high angles of attack $\left(\alpha=21.4^{\circ}\right.$ and $\left.15.0^{\circ}\right)$ only a small buckle is visible at the position where eventually the suction peak occurs for lower angles of attack $\left(\alpha=10.0^{\circ}, 5.0^{\circ}\right.$ and $\left.0.0^{\circ}\right)$. This is highlighted in figure 11(b) for angles of attack of $\alpha=21.4^{\circ}$ and $5.0^{\circ}$.

Having a closer look at the geometry of the leading edge region (figure 11(c)) it becomes apparent, that the pressure peak developes close to the lower slat attachment point (for retracted slat) on the lower surface of the airfoil. From this investigation it becomes evident, that at the two points where the slat trailing edges are located when the configuration is undeflected local pressure peaks can develop. Whereas the pressure peak on the upper surface kink is present for all angles of atttack (figure 11(a), at $x=0.19$ ), the pressure peak on the lower side only develops for lower angles of attack.

This behaviour can lead to a limitation of the transition prediction method, if the laminar boundary-layer profiles are calculated with the help of a boundary-layer code. Depending on the dimension of the suction peak, transition can be predicted too far upstream at the leading edge of the geometry for certain flow conditions. One idea to overcome this difficulty is to smooth the pressure distribution in the region near the leading edge to avoid an early laminar separation in the boundary-layer code. However, manipulating the pressure distribution in the leading edge region may have a considerable effect on the development of cross flow amplifications for general three-dimensional configurations.

On the one hand caution has to be paid when calculating polars for high-lift configurations at different angles of attack (or at relatively lower angles of attack). On the other hand, these problems do not arise when using the edge streamline approach, where boundary-layer data are directly extracted from the NavierStokes calculation. Firstly, no separation has been observed in the Navier-Stokes solution for all cases investigated here. Secondly, if separation would have been present, it is generally possible to do a stability analysis over small areas of separated flow with the edge streamline approach. But then, to capture all flow phenomena like for example cross flow instabilities, a much higher grid resolution of the boundary layer in the Navier-Stokes computation is needed.

\section{Application to general complex geometries}

Generally, the edge streamline approach with internally computed boundary-layer data (section II, C) can be applied in a very flexible way to geometries of different degree of complexity. Within its area of validity this method can be applied to abitrary geometries for example to high-lift wing systems, fuselages, arbitrarely shaped wings or nacelles. Limitations of the method exist mainly in areas, where it is not possible to determine the boundary-layer edge accurately or to extract boundary-layer profiles normal to

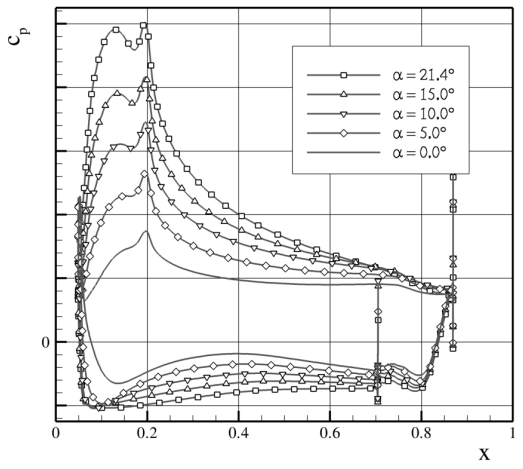

(a) Pressure distribution for main wing for $\alpha=$ $5.0^{\circ}$ to $21.4^{\circ}$

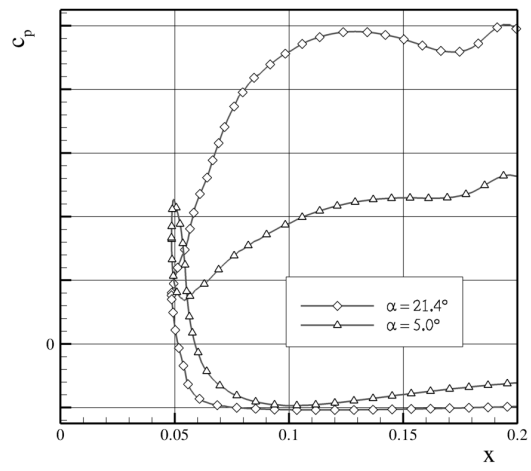

(b) Close-up of pressure distribution at leading edge of main airfoil for $\alpha=5.0^{\circ}$ and $21.4^{\circ}$.

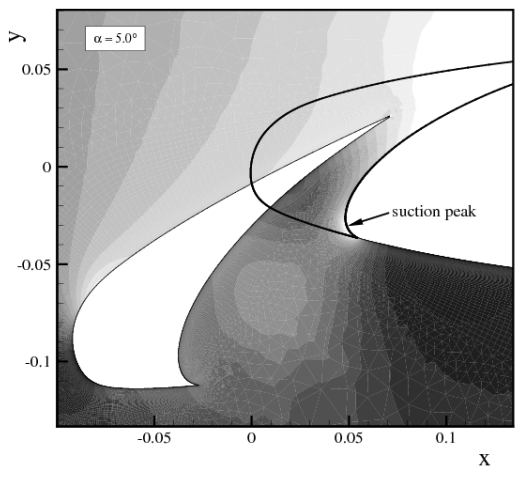

(c) Pressure contours at the leading edge of main airfoil for $\alpha=5.0^{\circ}$.

Figure 11. 2D high-lift configuration. 
the surface. This is, for instance, given in areas where two surface

parts intersect (for example wing-body junction). The resolution of the computational grids commonly used, especially in aircraft indutry, is not high enough to capture all flow features necessary for accurate transition prediciton with this method. Typically, the cross flow components of the velocity profiles inside the boundary layer are not accurately enough resolved for reliable prediction of the cross flow amplifications. This means, that the state of the art for transition prediction with the $e^{N}$-method is the use of a boundary-layer method to generate highly accurate boundary-layer data. This method is less flexible and much more restricted, depending on the boundary-layer method (here: swept, tapered wings) and needs very often more input information provided by the user. In the following some aspects are presented to facilitate the applicability beyond the general operative range.

Automatic DETECTION OF GEOMETRY VARIATIONS The boundary-layer code needs, together with certain on-flow conditions (Re, $M a$, angle of attack) and the pressure distribution, the geometrical sweep angles as input. The implementation of the transition module considers different ways to determine the leading edge and trailing edge sweep angles. One way is to prescribe the sweep angles. This can be done via user input globally for a set of line-in-flight cuts, usually covering the complete wing in spanwise direction, or separetly for each individual line-in-flight cut. Whereas the global prescription of the sweep angles does not account for variable sweep and exhibits therefore a certain limitation, the local prescription of the sweep angles requires a certain effort from the user in setting up the calculation. Additionaly to the manual prescription of the sweep angles, a method has been implemented to determine the local geometrical leading edge and trailing edge sweep angles automatically from the computational grid. This method uses only local data (to account for simple parallelization), this means, geometrical information is only gained from a certain surface grid point and its corresponding neighbour points.

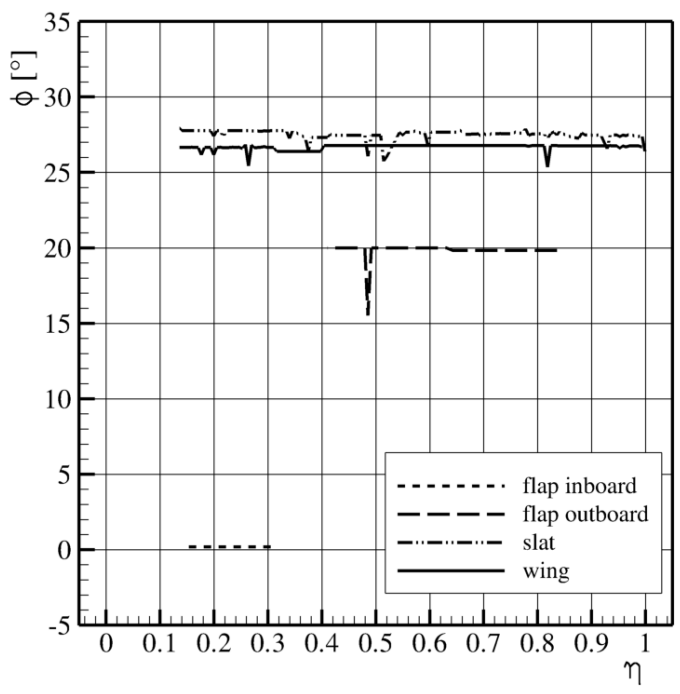

(a) Leading edge sweep angles.

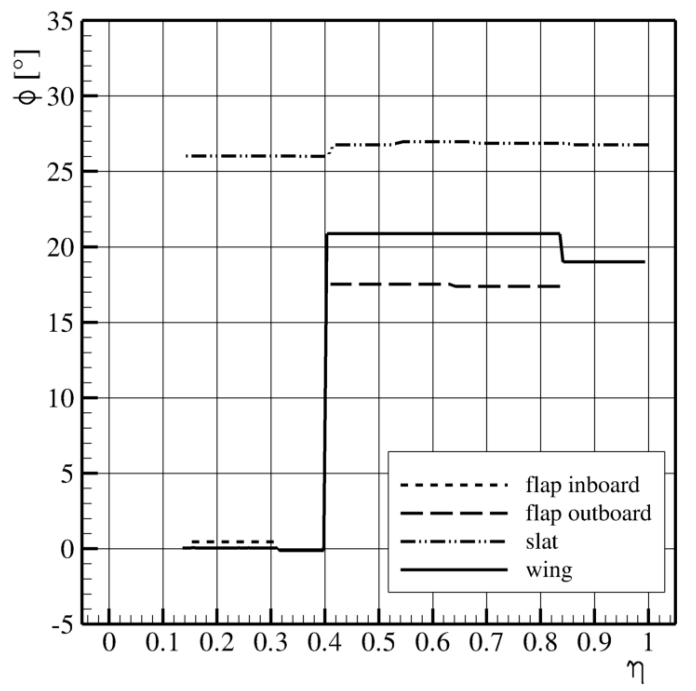

(b) Trailing edge sweep angles.

Figure 12. Spanwise sweep angle distribution for slat, wing and flap of a $3 \mathrm{D}$ high-lift configuration.

Figure 12 shows the spanwise variation of the automatically determined geometrical sweep angles for the 3D high-lift configuration presented in section 1. For this test case, a hybrid unstructured mesh was used with a surface representation of triangular cells. Despite of its very local definition, the automatic approach gives fairly accurate sweep angles for the trailing edges of slat, wing and flap (figure 12(b)). This is due to the fact, that the rear parts of the geometry are represented by a well defined edge and thus grid points are lying exactly on the trailing edge line. More uncertainties can arise for the local determination of the leading edge sweep angles. Here, the leading edge is usually not represented by grid lines when considering a surface grid with triangular cells. The local determination of the leading edge sweep angle is moreover influenced by the grid resolution: the coarser the grid the less accurate the representation of the original surface and, 
hence, the stronger the potential variation of the calculated sweep angles. This approach is very sensitive to local geometry variations. For this reason, certain small areas on the slat and the flap have been excluded from the treatment (figure 9(a)), where the surface is not continous (different slat and flap positions along the span). For the rest of the geometry, the local determination of the leading edge sweep mainly exhibits only variations less than $2.0^{\circ}$ (figure $12(\mathrm{a})$ ). However, a detailed analysis of the influence of the geometrical sweep angle on the boundary-layer calculation has yet to be done.

Another variation of the geometry that can be accounted for in the transition prediction module is that of the surface orientation. By individually defined cutting planes, line-in-flight cuts can be calculated for wings, vertical tail planes, winglets and wings or tail planes with distinct dihedral. The definition of the cutting plane can be given by the user globally for a set of line-in-flight cuts or for each line-in-flight cut seperately. Additionally, an automated approach can be used, that only needs a definition point on the surface of the geometry. At this point the surface normal vector is extracted and together with the vector of the incoming flow the normal vector defining the cutting plane is constructed. With this, a smooth adaptation of the line-in-flight cuts to the geometry is possible.

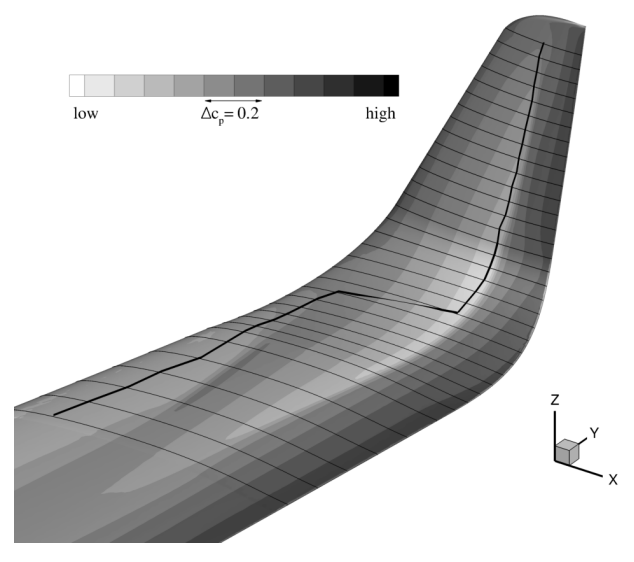

Figure 13. Line-in-flight cuts and predicted transition line at winglet of transport aircraft.

To verify this, transition has been predicted for the winglet of a 3D generic transport aircraft at $R e=3.0 \times 10^{6}, M a=0.78$, $\alpha=1.3^{\circ}$. The line-in-flight cuts used for transition prediction as well as the converged transition location are shown in figure 13.

ADAPTABLE TRANSITION LINES In order to predict transition automatically for three-dimensional aircraft configurations, the treatment at regions where the presented method leaves its area of validity has to be verified. Depending on the approach to determine the boundary-layer data (section II, C) the validity may be violated at wing tips or in the vicinity of junctions of different geometry parts. To account for these limitations, an approach to adapt the transition lines to the characteristics of the geometry has been implemented.

For the Navier-Stokes solver TAU in combination with the transition prediction module a transition line is specified for a certain boundary part. In the commonly used approach in the transition prediction module, this line is solely composed of the discrete transition points predicted for each transition prediction line. The approach to adapt the transition line to the geometry is to use a combined transition line, which is composed of points coming from the transition prediction and points prescribed by the user. To give an understanding of this approach, the procedure is demonstrated for the transition prediction on the lower wing of a 3D transport aircraft at $R e=3.0 \times 10^{6}, M a=0.78, \alpha=1.3^{\circ}$. The geometry consists of a fuselage and a wing, which is equipped with flap track fairings (figure 14).

At the beginning of the transition prediction a number of 'definition' points is prescribed. These definition points either serve as initial points for the line-in-flight cut or edge streamline calculation or as fixed transition points (filled symbols in figures 14(a) and 14(b)). For both types of definition points there exist two modes, giving four modes in total:

1. Completely fixed points (indicated as 'fixed' in figure 14).

2. Points that will be projected onto the connection line of the neighbour points if this connection line lies upstream of the point (indicated as 'upstream moveable' in figure 14).

3. Points, for which a transition prediction line will be calculate and for which the predicted transition location will be used (indicated as 'free' in figure 14).

4. Points, for which a transition prediction line will be calculate and for which the predicted transition location will be used, if this location is upstream of the definition point (indicated as 'tripped' in figure $14)$.

The effect of the different modes can be seen, if the transition prediction for this case either considers only Tollmien-Schlichting instabilites (figures 14(a) and 14(c)) or if Tollmien-Schlichting and cross flow 


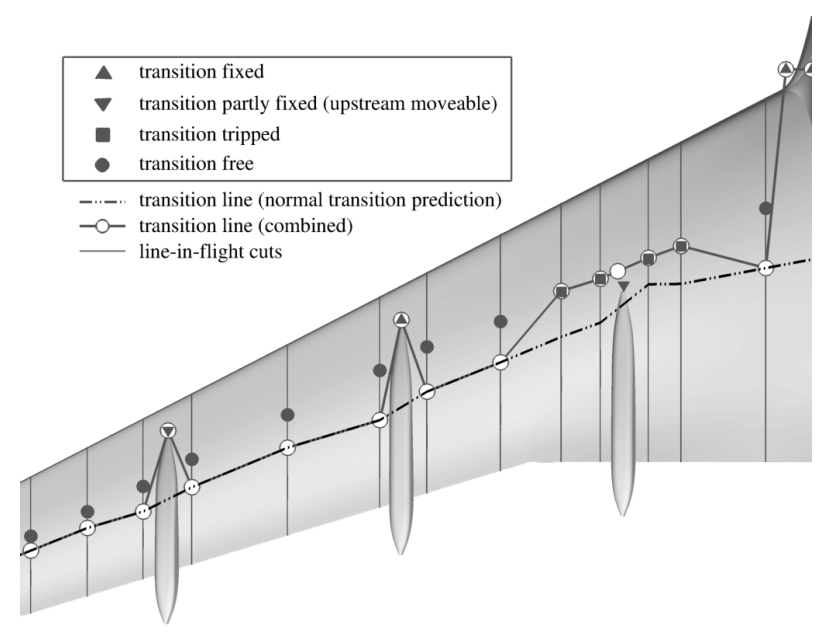

(a) Definition points and predicted transition lines. Only TS instabilities considered.

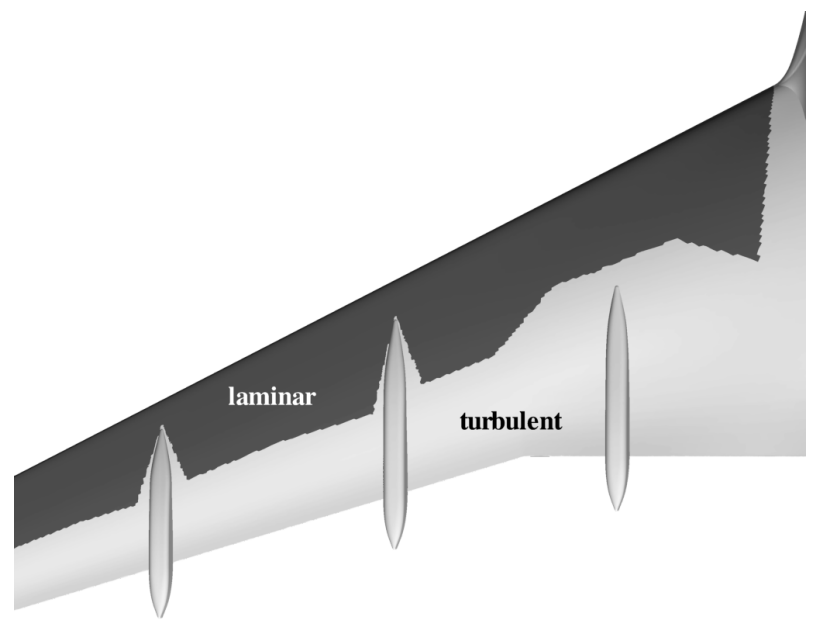

(c) Laminar and turbulent regions for adapted transition line (only TS).

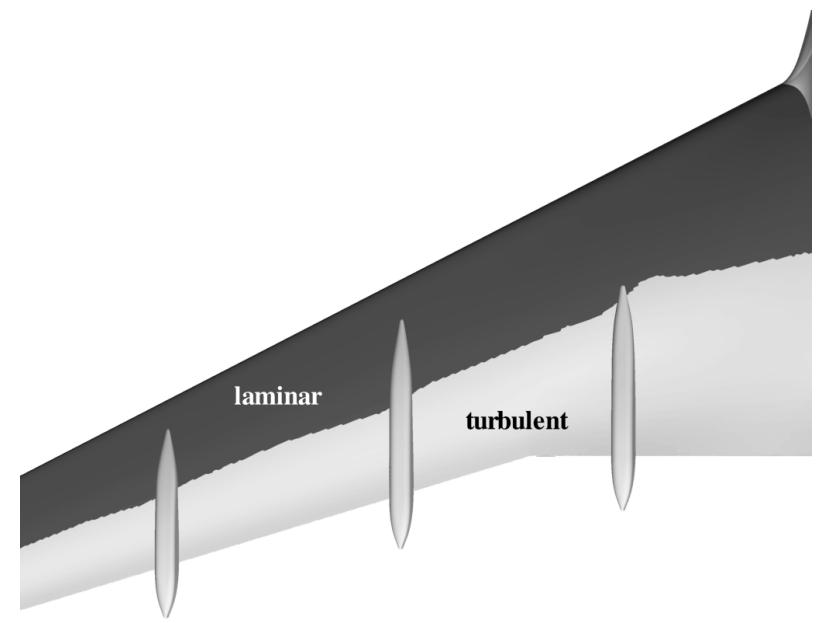

(e) Laminar and turbulent regions for non-adapted transition line(only TS).

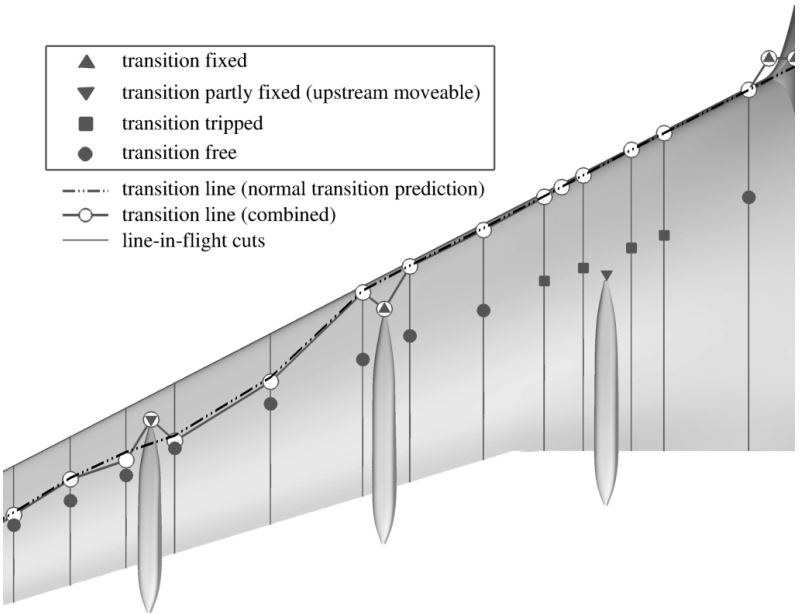

(b) Definition points and predicted transition lines.TS and CF instabilities considered.

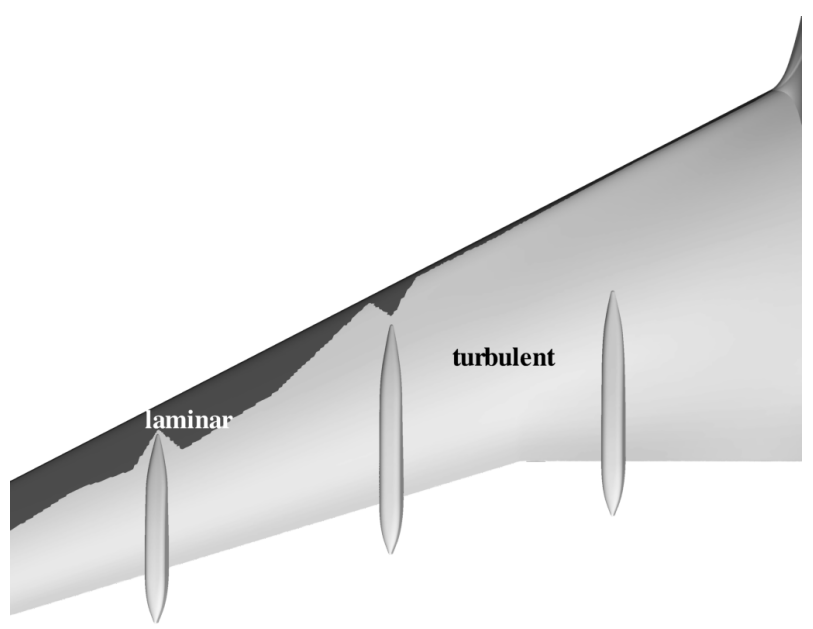

(d) Laminar and turbulent regions for adapted transition line (TS and CF).

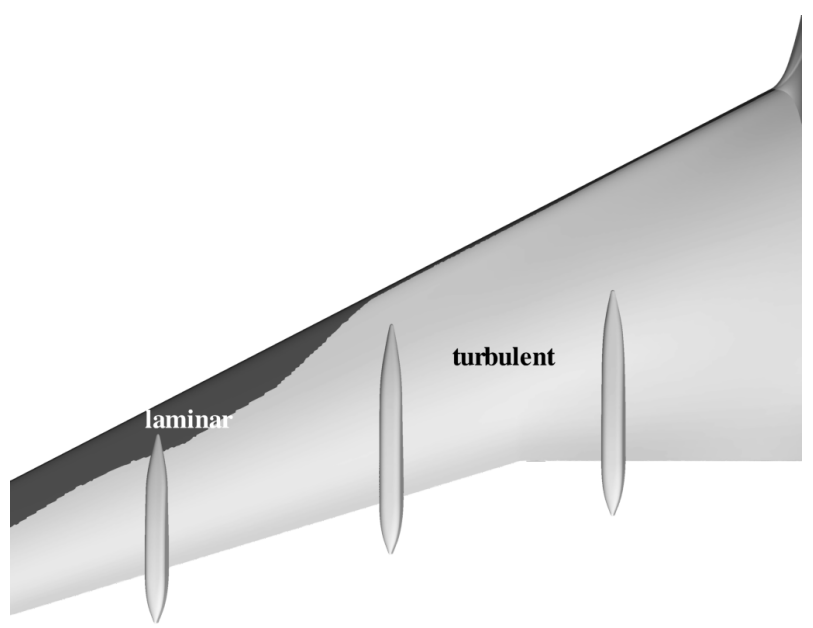

(f) Laminar and turbulent regions for non-adapted transition line ( $\mathrm{TS}$ and $\mathrm{CF}$ ).

Figure 14. Adapted transition lines for lower surface of a wing of 3D transport aircraft. 
instabilities are considered both (figures 14(b) and 14(d)). For comparison, the extent of the laminar and turbulent regions of common approach are visualized in figures 14(e) and 14(f).

At the wing body junction two fixed transition points have been placed upstream of the leading edge of the wing to simulate the turbulent boundary layer coming from the fuselage. The inboard flap track fairing is surrounded by tripped transition points and a partly fixed point for demonstration purposes. For the Tollmien-Schlichting case transition is predicted downstream of the transition tripping, hence the transition line in this region is composed of the initial definition points and the partly fixed point is projected upstream onto the transition line (figure 14(a)). For the combined Tollmien-Schlichting and cross flow case, transition is predicted in this region near the leading edge upstream of the tripping. This means, all points of the transition line are located at the predicted transition line (figure 14(b)). At the middle flap track fairing a fixed transitoin point is set to demonstrate the usefulness of the upstream projection of points. It is clearly visible, that for the cross flow case the fixed transition point leads to an unwanted behaviour since the transition obviouly would be upstream of the flap track fairing (figure 14(d)).

This test case shows the possibility to adapt the predicted transition line in various ways to account for example for turbulent wedges generated by defined obstacles on the surface or for turbulent flows generated at other parts of the geometry. Additionally, transition tripping as known from wind tunnel tests can also be accounted for.

\section{Conclusion}

The functionality of a transition prediction module attached to a Navier-Stokes solver is presented, with emphasis on the industrialization of the transition prediction procedure for the regular application to industrial relevant, three-dimensional configurations. The general approach of the presented work is to demonstrate the applicability of an automatic transition prediction in form of the $e^{N}$-method to complex configurations and fully three-dimensional boundary layers.

Two different approaches to determine the boundary-layer data needed for the stability analysis and to apply the transition criterion in form of the $e^{N}$-method are presented. One approach is to apply a boundary layer method along line-in-flight cuts, the other approach is to extract laminar boundary layer data directly from the Navier-Stokes solution along edge streamlines.

The parallelization of the transition module is described and the parallel and computational performance of the method is presented by means of two different three-dimensional test cases.

For the flow around an inclined prolate spheroid, it is shown that the two $N$-factor $e^{N}$-method with boundary layer data from a Navier-Stokes solution is applicable for the prediction of transition of threedimensional boundary layer flows. The validation study shows a good agreement of the numerically predicted transition lines with experimental data.

The flow around different three-dimensional configurations is investigated to show the feasibility of the presented method to industrial relevant, complex aircraft geometries. Different approaches for the industrialization of the transition prediction module are presented and strategies for the application of the method for complex configurations are given.

\section{Acknowledgments}

For the different three-dimensional transport aircraft configurations presented, computational grids and restart solutions have been provided by Martin Kruse, Jan Himisch and Stefan Melber-Wilkending of DLR, German Aerospace Center, Braunschweig, Germany. This support is gratefully acknowledged. The authors thank Airbus for letting them use the geometry and computational grid for the 2D A310 take-off configuration.

\section{References}

${ }^{1}$ Langtry, R. B., Menter, F. R., Likki, S. R., Suzen, Y. B., Huang, P. G., and Völker, S., "A Correlation-Based Transition Model Using Local Variables - Part II: Test Cases and Industrial Applications," Journal of Turbomachinery, Vol. $128,2006$.

${ }^{2}$ Menter, F. R., Langtry, R. B., Likki, S. R., Suzen, Y. B., Huang, P. G., and Völker, S., "A Correlation-Based Transition Model Using Local Variables - Part I: Model Formulation," Journal of Turbomachinery, Vol. 128, 2006.

${ }^{3}$ Smith, A. M. O. and Gamberoni, N., "Transition, Pressure Gradient and Stability Theory," Tech. rep., 1956, Douglas Aircraft Company Inc., El Segundo, California, U.S.A, Report ES 26388. 
${ }^{4}$ van Ingen, J. L., "Een semi-empirische methode voor de bepaling van de ligging van het omslaggebied van de greenslaag bij onsamedrukbare tweedimensionale stromingen," Tech. rep., 1956, Technische Hogeschool Vliegtuigbouwkunde Delft, Nederland, Rapport V.T.H.-71.

${ }^{5}$ Schrauf, G., Perraud, J., and Lam, F., "Comparison of Boundary-Layer Transition Predictions Using Flight Test Data," Journal of Aircraft, Vol. 35, No. 6, 1998.

${ }^{6}$ Schrauf, G., "Large-Scale Laminar-Flow Tests Evaluated with Linear Stability Theory," Journal of Aircraft, Vol. 41, No. 2, 2004.

${ }^{7}$ Radespiel, R., Windte, J., and Scholz, U., "Numerical and Experimental Flow Analysis of Moving Airfoils with Laminar Separation Bubbles," AIAA Journal, Vol. 45, No. 6, 2006, pp. 1346-1356.

${ }^{8}$ Wokoeck, R., Krimmelbein, N., Ortmanns, J., Ciobaca, V., Radespiel, R., and Krumbein, A., "RANS Simulation and Experiments on the Stall Behaviour of an Airfoil with Laminar Separation Bubbles," 44th AIAA Aerospace Sciences Meeting and Exhibit, AIAA Paper 2006-244, Reno, 2006.

${ }^{9}$ Windte, J., Scholz, U., and Radespiel, R., "Validation of the RANS-Simulation of Laminar Separation Bubbles on Airfoils," Aerospace Science and Technology, Vol. 10, No. 6, 2006, pp. 484-494.

${ }^{10}$ Krumbein, A., "Automatic Transition Prediction for High-Lift Systems Using a Hybrid Flow Solver," Journal of Aircraft, Vol. 42, 2005, pp. 1362-1366.

${ }^{11}$ Krumbein, A., "Automatic Transition Prediction and Application to Three-Dimensional Wing ConfiguraÂtions," Journal of Aircraft, Vol. 44, No. 1, 2007, pp. 119-133.

${ }^{12}$ Krumbein, A. and Krimmelbein, N., "Navier-Stokes High-lift Airfoil Computations with Automatic Transition Prediction Using the DLR TAU Code," New Results in Numerical and Experimental Fluid Mechanics VI. Contributions to the 15th STAB/DGLR Symposium Darmstadt, edited by C. Tropea, S. Jakirlic, H. J. Heinemann, R. Henke, and H. Hönlinger, Springer, Berlin Heidelberg, 2007, ISBN 978-3-540-74458-0.

${ }^{13}$ Toulorge, T., Ponsin, J., Perraud, J., and Moens, F., "Automatic Transition Prediction for RANS Computations Applied to a Generic High-Lift Wing," 45th AIAA Aerospace Sciences Meeting and Exhibit, AIAA Paper 2007-1086, Reno, 2007.

${ }^{14}$ Stock, H. W., " $e^{N}$ Transition Prediction in Three-Dimensional Boundary Layers on Inclined Prolate Spheroids," AIAA Journal, Vol. 44, No. 1, 2006, pp. 108-118.

${ }^{15}$ Arthur, M. T. and Atkin, C. J., "Transition modelling for viscous flow prediction," 36th AIAA Fluid Dynamics Conference and Exhibit, AIAA Paper 2006-3052, San Francisco, 2006.

${ }^{16}$ Krimmelbein, N., Radespiel, R., and Nebel, C., "Numerical Aspects of Transition Prediction for Three-Dimensional Configurations," 35th AIAA Fluid Dynamics Conference and Exhibit, AIAA Paper 2005-4764, Toronto, 2005.

${ }^{17}$ Krumbein, A., Krimmelbein, N., and Schrauf, G., "Application of a Hybrid Navier-Stokes Solver with Automatic Transition Prediction," 25th AIAA Applied Aerodynamics Conference, AIAA Paper 2007-4284, Miami, 2007.

${ }^{18}$ Schwamborn, D., Gerhold, T., and Hannemann, V., "On the Validation of the DLR-TAU Code," New Results in Numerical and Experimental Fluid Mechanics, edited by W. Nitsche, H.-J. Heinemann, and R. Hilbig, No. 72, Vieweg, 1999, pp. 426-433, ISBN 3-528-03122-0.

${ }^{19}$ Schwamborn, D., Gerhold, T., and Heinrich, R., "The DLR TAU-Code: Recent Applications in Research and Industry," European Conference on Computational Fluid Dynamics. ECCOMAS CFD 2006, edited by P. Wesseling, E. Onate, and J. Periaux, 2006.

${ }^{20}$ Schrauf, G., "COCO - A program to compute velocity and temperature profiles for local and nonlocal stability analysis of compressible, conical boundary layers with suction," Tech. rep., 1998, ZARM Technik Report.

${ }^{21}$ Schrauf, G., "LILO 2.1. User's Guide and Tutorial," Tech. rep., 2006, GSSC Technical Report 6, originally issued Sep. 2004, modified for Version 2.1.

22 "MPI: A message-passing interface standard 1.0. Technical report," http://www.mpi-forum.org, 1994.

${ }^{23}$ Schrauf, G., "Transition prediction using different linear stability analysis strategies," 12th AIAA Applied Aerodynamics Conference, AIAA Paper 1994-1848, Colorado Springs, 1994.

${ }^{24}$ Gaster, M., "A note on the relation between temporally increasing and spatially increasing disturbances in hydrodynamic stability," J. Fluid. Mech., Vol. 14, 1962.

${ }^{25}$ Kaups, K. and Cebeci, T., "Compressible Laminar Boundary Layers with Suction on Swept and Tapered Wings," Journal of Aircraft, Vol. 14, No. 7, 1977.

${ }^{26}$ Hirschel, E. H., Basics of Aerothermodynamics, Springer, Berlin Heidelberg, 2005, p. 201, ISBN 978-3-540-26519-1.

${ }^{27}$ Krumbein, A., Krimmelbein, N., and Schrauf, G., "Automatic Transition Prediction in a Hybrid Flow Solver - Part 1: Methodology and Sensitivities," Journal of Aircraft, Vol. 46, No. 4, 2009, pp. 1176-1190.

${ }^{28}$ Krumbein, A., Krimmelbein, N., and Schrauf, G., "Automatic Transition Prediction in a Hybrid Flow Solver - Part 2: Practical Application," Journal of Aircraft, Vol. 46, No. 4, 2009, pp. 1191-1199.

${ }^{29}$ Krimmelbein, N. and Radespiel, R., "Transition prediction for three-dimensional flows using parallel computation," Computers \& Fluids, Vol. 38, 2009, pp. 121-136.

${ }^{30}$ Krumbein, A., " $e^{N}$ Transition Prediction for 3D Wing Configurations using Database Methods and a local, linear Stability Code," Aerospace Science and Technology, Vol. 12, No. 8, 2008, pp. 592-598.

${ }^{31}$ Kreplin, H.-P., Meier, H. U., and Maier, A., "Wind Tunnel Model and Measuring Techniques for the Investigation of Three-Dimensional Turbulent Boundary Layers," 10th AIAA Aerodynamic Testing Conference, AIAA Paper 78-781, San Diego, 1978.

${ }^{32}$ Kreplin, H.-P., Vollmers, H., and Meier, H. U., "Wall Shear Stress Measurements on an Inclined Prolate Spheroid in the DFVLR 3m x 3m Low Speed Wind Tunnel, Göttingen," Tech. rep., 1985, DFVLR-AVA, Report IB 22-84 A 33.

${ }^{33}$ Melber-Wilkending, S., "Aerodynamics of the Wing/Fuselage Junction at an Transport Aircraft in High-lift Configuration," 16th AG STAB/DGLR Symposium, 2008.

${ }^{34}$ Manie, F., Piccin, O., and Ray, J., "Test Report of the 2D Model M1 in the ONERA F1 Wind Tunnel," GARTEUR $\mathrm{AD}(\mathrm{AG}-08), \mathrm{TP}-041,1989$.

16 of 16 